\title{
The Effect of Hyaluronic Acid in Preventing Recurrence of Anterior Urethral Stricture after Endoscopic Internal Urethrotomy- A Single Centre, Randomised Controlled, Single Blind Study
}

\author{
Chikamoga Siddaiah Manohar ${ }^{1}$, Vinish Kumar Singh², Ramaiah Keshavamurthy ${ }^{3}$, Jayaram Sreenivas ${ }^{4}$, Avneet Gupta ${ }^{5}$, \\ Sharana Bassapa Rudrawadi ${ }^{6}$ \\ ${ }^{1}$ Department of Nephrology, Institute of Nephro Urology Affiliated to Rajiv Gandhi University of Health Sciences, \\ Bangalore, Karnataka, India. ${ }^{2}$ Department of Nephrology, Institute of Nephro Urology Affiliated to Rajiv Gandhi \\ University of Health Sciences, Bangalore, Karnataka, India. ${ }^{3}$ Department of Nephrology, Institute of Nephro Urology \\ Affiliated to Rajiv Gandhi University of Health Sciences, Bangalore, Karnataka, India. ${ }^{4}$ Department of Nephrology, \\ Institute of Nephro Urology Affiliated to Rajiv Gandhi University of Health Sciences, Bangalore, Karnataka, India. \\ ${ }^{5}$ Department of Nephrology, Institute of Nephro Urology Affiliated to Rajiv Gandhi University of Health Sciences, \\ Bangalore, Karnataka, India. ${ }^{6}$ Department of Nephrology, Institute of Nephro Urology Affiliated to Rajiv Gandhi \\ University of Health Sciences, Bangalore, Karnataka, India.
}

\section{ABSTRACT}

\section{BACKGROUND}

Hyaluronidase instillation during OIU may decrease the incidence of urethral stricture recurrence. ${ }^{13}$ The exact mechanism is not known in urethral stricture, but it is used as antifibrotic agent in hypertrophic scar, keloid, and pulmonary fibrosis. Intralesional injection decreases fibroblast proliferation, collagen, and glycosaminoglycan synthesis and suppresses proinflammatory mediators in wound healing process. ${ }^{14}$ This study was conducted to see the benefits of HA for preventing recurrence of anterior urethral stricture after urethrotomy.

\section{METHODS}

After obtaining the clearance from ethical committee, 70 patients satisfying the inclusion criteria were recruited \& randomly divided into 2 groups: Group A (35 patients, experimental group) received hyaluronic acid (HA) instillation \& Group B (35 patients, Control group) received lubricant lignocaine after EIU. Each patient was evaluated at 4 weeks (V1), 12 weeks (V2), \& 24 weeks (V3) after surgery. The recurrence rate was evaluated by protocol analysis based on number of patients who completed the study. Preoperative characteristics, including the site \& length of urethral stricture, were evaluated by intent-to-treat analysis.

\section{RESULTS}

Among 70 patients, only 30 patients in group A \& 30 patients in group B had completed the study. Significant improvement noted in both groups after surgery in every parameter with recurrence in Group A 9.43\% \& in Group B 22.92\% (p <0.05) at V3. IPSS score at V3 were obstructive: Group A - 4.03 \pm 0.65 , Group B $-5.16 \pm 1.84$ $(p=0.0024)$, Irritative: group A $-2.8 \pm 0.60$, Group B $-3.33 \pm 1.19(p=0.033)$. At V3 follow up Qmax: Group A - 22.40 \pm 3.21 , Group B - 18.13 $\pm 3.56(p=0.0001)$; Qavg: Group A - 11.43 \pm 1.72 , Group B - 10.43 \pm 2.2 ( $\mathrm{p}=0.048$ ); PVRV: Group A - $10.16 \pm$ 13.81, Group B - 19.33 \pm 15.90 ( $p=0.020$ ); Degree of satisfaction: Group A $-2.11 \pm$ 0.38 , Group B - $1.76 \pm 0.49$ ( $\mathrm{p}=0.0031)$.

\section{CONCLUSIONS}

HA instillation during EIU decreases the incidence of urethral stricture recurrence \& improves degree of satisfaction in long term follow up without any side effect.
Corresponding Author:

Dr. Sushreesmita Mohanty,

Associate Professor,

Department of

Hi-Tech Medial

Odisha, India.

E-mail: drsushreesmita98@gmail.com

DOI: $10.14260 /$ jemds/2020/10

Financial or Other Competing Interests: None.

How to Cite This Article:

Manohar CS, Singh VK, Keshavamurthy $R$, et al. The effect of hyaluronic acid in preventing recurrence of anterior urethral stricture after endoscopic internal urethrotomy- a single centre, randomised controlled, single blind study. J. Evolution Med. Dent. Sci. 2020;9(01):44-48, DOI: 10.14260/jemds/2020/10

Submission 30-10-2019,

Peer Review 21-12-2019,

Acceptance 27-12-2019,

Published 06-01-2020.

\section{KEY WORDS}

Urethra, Recurrence, Voiding 


\section{BACKGROUND}

Male urethral stricture disease continues to be a common and challenging urological condition ${ }^{1-4}$. Despite the high failure rate of directly visualized internal urethrotomy (DVIU), it remains the most common performed procedure for the treatment of anterior urethral strictures. ${ }^{5-7}$ Even when DVIU is performed in short bulbar stricture $(<2 \mathrm{~cm})$, recurrence rate at 12 months was approximately $40 \%{ }^{8-10}$ Repeated DVIU had even more recurrence rate and increased stricture complexity, stricture length. To reduce recurrence rate, Laser surgery, self- catheterization after DVIU, application of converting enzyme inhibitor gels, and steroid injection have been used. ${ }^{8-11}$ Several materials have been employed in that prevent postoperative adhesion, including hyaluronic acid (HA), carboxymethylcellulose (CBC), Mitomycin C, Triamcinolone and their combinations. Hyaluronidase instillation during DVIU may decrease the incidence of urethral stricture recurrence. ${ }^{8}$ The anionic polysaccharide HA is a major constituent of extracellular matrix and is thus found in the connective tissue, skin, cartilage and synovial fluid. It is a hydrophilic and non-immunogenic macro molecule that easily quotes and lubricate mucus membrane. These physical properties of HA unable it to reduce or prevent trauma at the site of surgery. ${ }^{12}$ Hyaluronidase instillation during OIU may decrease the incidence of urethral stricture recurrence. ${ }^{13}$ The exact mechanism is not known in urethral stricture but it is used as antifibrotic agent in hypertrophic scar, keloid, and pulmonary fibrosis. Intralesional injection decreases fibroblast proliferation, collagen, and glycosaminoglycan synthesis and suppresses proinflammatory mediators in wound healing process. ${ }^{14}$ This study was conducted to see the benefits of HA for preventing recurrence of anterior urethral stricture after urethrotomy.

\section{METHODS}

A total of 70 patients who were passed the inclusion criteria and treated for urethral stricture disease by DVIU between Jan 2016 to Jan 2017 were invited to participate in the present study after taking informed consent. This study is single center, randomized controlled, single blind. The study was approved by institution review board.

Inclusion criteria were (1) a urethral stricture observed by retrograde urethrography, (2) Primary strictures, (3) Short segment $(<2.5 \mathrm{~cm})$, (4) less degree of spongio fibrosis, (5) patients who was willing and able to participate in the present clinical study. Patients who (1) had undergone EIU in the past to treat urethral stricture, (2) had undergone any other transurethral surgeries, (3) had undergone radical prostatectomy for prostate cancer, (4) had $\geq 2.5 \mathrm{~cm}$ or more urethral stricture, (5) had a neurogenic bladder, or (6) had a urinary tract infection were excluded from the study.

Patients who met the inclusion criteria were randomized by simple block randomization to either (1) experimental group (Group A, 35 patients who received HA instillation), or the control group (Group B, who received and lubricant instillation) after DVIU.
The primary end point was the recurrence of urethral stricture 24 week after surgery. The secondary endpoints were voiding symptoms and degree of satisfaction.

At beginning of the study (V0), information regarding the duration of illness and the medical history was collected. Retrograde urethrography (RGU) and ultrasound (USG) were also performed during V0 to measure the length, site of stricture and degree of spongiofibrosis. Patients were evaluated at 4 weeks (V1), 12 weeks (V2), and 24 weeks (V3) after surgery based on the International Prostate symptoms score, Peak urinary flow rate(Qmax),average flow rate (Qavg), voided volume, post void residual volume and degree of satisfaction. Failure was defined as the need to repeat surgical intervention due to lack of improvement in voiding symptoms. In addition, failure was confirmed by retrograde urethrography. Constructive uroflow curves or a maximum flow rate $<10 \mathrm{ml} / \mathrm{sec}$, as determined by uroflowmetry, were considered to indicate recurrence. Recurrence in patients with constructive flow rate or $\mathrm{Qmax}<10 \mathrm{ml} / \mathrm{sec}$ confirmed by RGU and Cystoscopy.

\section{Surgical Techniques}

The patient was placed under spinal anaesthesia and EIU was performed at the 12 o'clock position by using a 21 Fr. Storz urethrotome with a straight cold knife. After urethrotomy, and 16 Fr Foley catheter as inserted. Upon pulling the Foley to block the bladder neck, $5 \mathrm{mg}$ of HA or $5 \mathrm{mg}$ of lubricant (Lignocaine) as instilled between the urethral lumen and the Foley catheter. After instillation, gauze was used to maintain the traction of the Foley catheter, which enabled to blockade the bladder neck. One day after surgery, gauze traction was removed. The Foley catheter was removed 1 weeks after EIU.

\section{Statistical Analysis}

The recurrence rate was evaluated by per protocol analysis based on the number of patients who completed the study. Preoperative characteristics, including the site and length of urethral stricture, was evaluated by intent-to-treat analysis. Voiding symptoms, Qmax and PVRV, was compared by using the student's paired t-test. The chi-square test was used to analyse the recurrence rate, causes, and locations of urethral stricture. The patients who was have no recurrence will compared to those who was have recurrence in terms of voiding symptoms, pain scale, and degree of satisfaction. SPSS software v.18.0 was used for statistical analysis, and a p-value $<0.05$ was considered to be statistically significant.

\section{Assessment of Efficacy and Safety}

To evaluate the ability of HA to prevent urethral stricture recurrences, the International Prostate Symptom Score (IPSS), peak urine flow rate (Qmax),Average Flow rate (Qavg) and post voiding residual urine volume (PVRV) were determined at every visit Retrograde urethrography was performed routinely at V2. The degree of patient satisfaction was recorded on a scale ranging from 0 (extremely satisfied) to 3 (extremely dissatisfied). The visual analogue scale (VAS) pain score was assessed on a 10-point Likert scale and the Quality of Life (QoL) score was also determined. The safety of HA treatment was assessed at V1, V2, and V3 on the basis of the patient's history, a physical examination, and the presence or absence of adverse effects. 


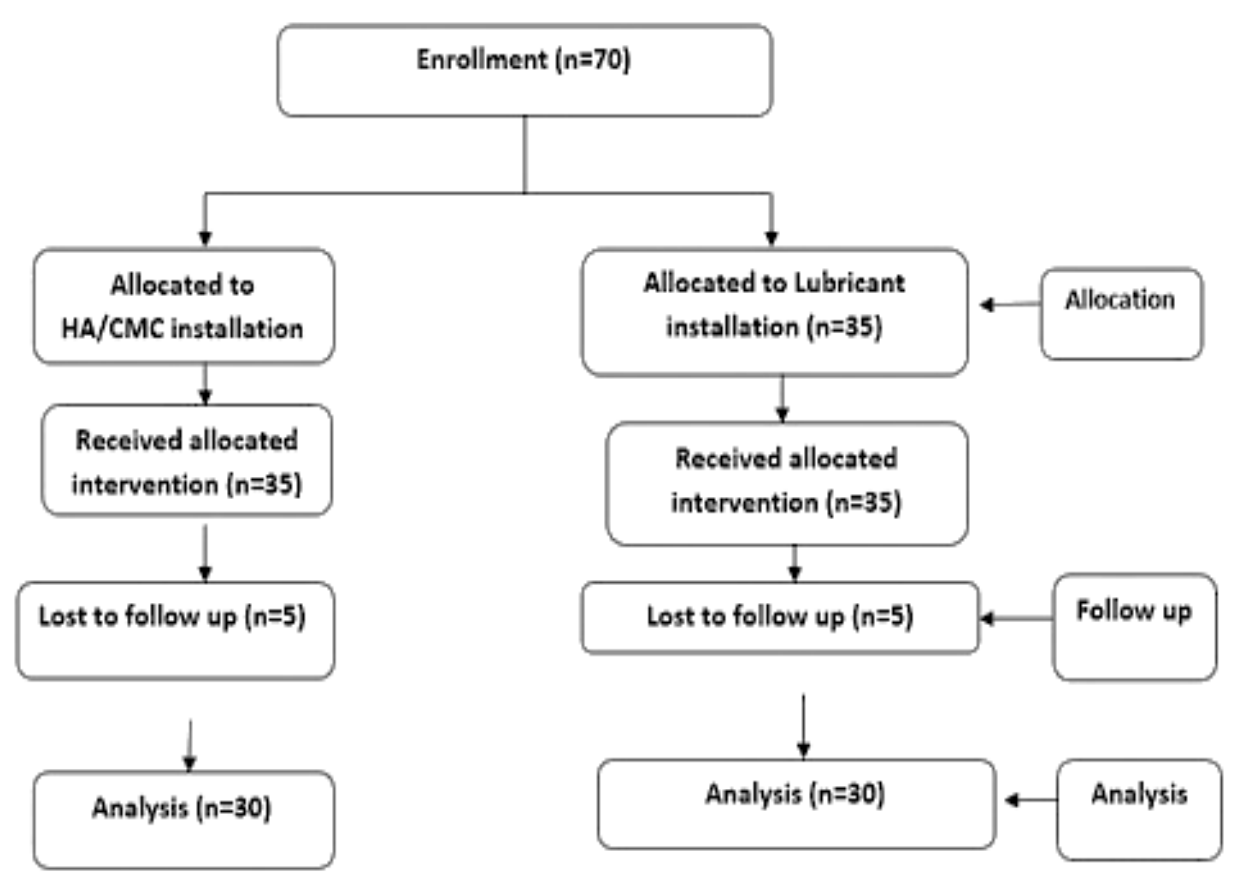

Figure 1. Postoperative Outcomes and Follow Up Analysis

\section{RESULTS}

\begin{tabular}{|c|c|c|c|}
\hline & Group A $(n=35)$ & Group B $(n=35)$ & p-Value \\
\hline Age, Years & $58.63 \pm 15.97$ & $54.57 \pm 18.69$ & 0.337 \\
\hline BMI, $\mathrm{Kg} / \mathrm{m}^{2}$ & $25.42 \pm 2.28$ & $25.93 \pm 2.87$ & 0.423 \\
\hline Stricture length, $\mathrm{cm}$ & $1.95 \pm 0.48$ & $1.75 \pm 0.43$ & 0.094 \\
\hline \multicolumn{4}{|l|}{ Stricture, location \%: } \\
\hline 1. bulbar & 62.26 & 64.58 & 0.957 \\
\hline 2. penile & 33.96 & 31.25 & \\
\hline 3. both & 3.78 & 4.17 & \\
\hline \multicolumn{4}{|l|}{ Stricture, Causes \% } \\
\hline 1. Trauma & 33.96 & 31.25 & 0.956 \\
\hline 2. Infection & 11.32 & 12.5 & \\
\hline 3. Iatrogenic & 16.98 & 20.83 & \\
\hline 4. Unknown & 37.74 & 35.42 & \\
\hline \multicolumn{4}{|l|}{ PRE OP IPSS } \\
\hline 1. Obstructive sub score & $16.26 \pm 2.2$ & $16.59 \pm 1.86$ & 0.559 \\
\hline 2. Irritative sub score & $11.63 \pm 1.47$ & $12.03 \pm 1.19$ & 0.251 \\
\hline QoL & $3.74 \pm 1.05$ & $3.70 \pm 1.18$ & 0.870 \\
\hline Qmax,ml/sec & $6.71 \pm 2.53$ & $7.56 \pm 1.70$ & 0.132 \\
\hline Qav & $2.96 \pm 1.67$ & $3.06 \pm 1.43$ & 0.804 \\
\hline PVRV, ml & $156.83 \pm 51.34$ & $145.12 \pm 52.36$ & 0.385 \\
\hline
\end{tabular}

\begin{tabular}{|c|c|c|c|c|}
\hline & & Group A & Group B & p Value \\
\hline $4^{\text {th }}$ Weeks & Obstructive IPSS & $0.3 \pm 0.77$ & $3.3 \pm 0.64$ & 0.1002 \\
\hline & Irritative IPSS & $1.66 \pm 0.64$ & $1.86 \pm 0.61$ & 0.2203 \\
\hline & Qmax & $27.43 \pm 4.32$ & $26.43 \pm 2.74$ & 0.288 \\
\hline & Qavg & $13.70 \pm 0.93$ & $13.40 \pm 0.84$ & 0.195 \\
\hline & PVRV & $10.14 \pm 11.80$ & $15.40 \pm 12.30$ & 0.080 \\
\hline $12^{\text {th }}$ weeks & Obstructive IPSS & $3.63 \pm 0.70$ & $4.1 \pm 0.90$ & 0.027 \\
\hline & Irritative IPSS & $2.43 \pm 0.55$ & $2.56 \pm 0.61$ & 0.389 \\
\hline & Qmax & $24.83 \pm 2.43$ & $24.16 \pm 2.16$ & 0.263 \\
\hline & Qavg & $12.23 \pm 1.82$ & $11.56 \pm 1.62$ & 0.138 \\
\hline & PVRV & $10.16 \pm 12.25$ & $17.25 \pm 14.40$ & 0.062 \\
\hline $24^{\text {th }}$ weeks & Obstructive IPSS & $4.03 \pm 0.65$ & $5.16 \pm 1.84$ & 0.002 \\
\hline & Irritative IPSS & $2.8 \pm 0.60$ & $3.33 \pm 1.19$ & 0.033 \\
\hline & Qmax & $22.40 \pm 3.21$ & $18.13 \pm 3.56$ & 0.001 \\
\hline & Qavg & $11.43 \pm 1.72$ & $10.40 \pm 2.2$ & 0.048 \\
\hline & PVRV & $10.16 \pm 13.81$ & $19.33 \pm 15.90$ & 0.020 \\
\hline Recurrence & \multicolumn{5}{|c|}{$5(09.43)$} & $11(22.92)$ & 0.029 \\
\hline Failure & \multicolumn{5}{|c|}{$6(10.00)$} & $7(11.67)$ & 0.473 \\
\hline \multicolumn{5}{|c|}{ Table 2. Surgical Outcomes } \\
\hline
\end{tabular}

Analysis of the intention-to-population revealed that group A and B did not differ significantly in terms of the location and length of urethral stricture (table 1).

Five group A (HA) and 5 group B (lubricant) patients were lost to follow- up. Thus, 30 of group A and group B patients completed the follow - up period. As shown in Table 1, six group. A (11.32\%) and seven group B subjects $(14.58 \%)$ showed no improvement of symptoms, which was interpreted as failure of the surgery $(p=0.473)$ Urethral stricture recurrence was observed in five group. A (9.4\%) and 11 group B cases $(22.9 \%)(p=0.029)$ (Table 1). Table 2 showed the baseline characteristics of patients deemed to have experienced surgical failure and recurrence groups. The urethrogram performed at V2 showed that patients who did not experience recurrence or failure had no narrowing.

\section{DISCUSSION}

The present study showed that the intraurethral instillation of HA during EIU significantly reduced the recurrence of urethral stricture after EIU. Moreover, 24 weeks after surgery, the experimental group had a better voiding symptom than the control group. Thus, HA was effective. It also had no noticeable side effects and helped to lessen the pain after surgery.

EIU is the primary treatment option for urethral stricture because of its simplicity and effectiveness. However, the success rate of EIU is low (about 40\%) and the recurrence rate is high ${ }^{15}$. Although the mechanisms behind urethral stricture recurrence after EIU are not yet understood clearly, fibrosis and adhesion of epithelial and sub epithelial spongy tissues during healing are likely to be involved ${ }^{16}$ HolmNielsen et al. reported that the post-EIU recurrence rate of urethral stricture was $23 \%-80 \%{ }^{17}$ In contrast, the recurrence rate of the control group in the present study was 
$22.9 \%$. This disparity may reflect the fact that the present study had a shorter follow up period than the previous study Nevertheless, compared to the control group in our study, the post- EIU recurrence rate of urethral stricture in the experimental group was significantly reduced $(9.4 \%)$.

Of the many techniques that have been used to reduce the recurrence rate, laser has several advantages over conventional surgery, including the ability to control bleeding (Which improves working visibility) and the ability to remove fibrotic tissues by vaporization during incision of the urethra. However, despite laser being associated with a high success rate and a low recurrence rate, laser does not differ significant form conventional methods in terms of long- term out comes. 8 \&18

Postoperative self- catheterization is sometimes suggested as a method that can reduce the recurrence rate. However, one study found that it did not significantly reduce the recurrence of urethral stricture as compared to the control group. With regard to other methods that may reduce recurrence, use of an antifibrotic angiotensin-converting enzyme - inhibitor gel after performing EIU has been suggested to reduce urethral stricture recurrence possibly by inhibiting fibrosis. In addition, injection of a steroid at the stricture point during EIU was recently reported to postpone, but not reduce, urethral stricture recurrence. The physiological properties of corticosteroids suggest that they may decrease scar formation by reducing the synthesis of collagen, glycosaminoglycans, and inflammatory mediators. ${ }^{19}$ (Table 3).

Extracellular matrix regulation plays a prominent role in this process, and it has been reported that sodium hyaluronate may reduce unwanted scar tissue by $50 \%$ or more. ${ }^{20}$ Moreover, histological analysis in an animal study by Hong et al. revealed that the group treated with HA/CMC exhibited fewer fibrous tissue attachments at the adhesion tissue after surgery. ${ }^{21}$ In the human body, several natural barriers, including the peritoneum, omentum, and amnion, serve to prevent adhesion between neighbouring tissues, when surgery breaches these barriers, a synthetic physical barrier can be introduced in separate the surface of injured tissue from the surrounding tissues, thereby reducing adhesion of the injured tissues to the nearby organs. HA is a fluid synthetic sol-gel barrier with a viscosity of 2500-3500 $\mathrm{CP}$, similar to that of honey In the present study, the idea to use HA/CMC to reduce post EIU urethral stricture recurrence was based on previous studies showing that the instillation of synthetic solution as tissue barriers during EIU inhibits scar formation.

In the present study, the patients who did not have stricture recurrence had significantly better Qmax values, IPSS obstructive subscores, and postoperative pain. HA/CMC effectively coats the wound surfaces and maintains the activity for a long time because of its high viscosity. HA installation during EIU may reduce postoperative urethral narrowing because it can prevent fibrosis and scar formation during healing of the urethrotomy wound. The fact that the patients treated with high-viscosity HA experienced significantly less postoperative pain than the lubricanttreated patients can be attributed to the extended presence of a lubricating barrier between the urethra, urethrotomy wounds, and the Foley's catheter.
Many different lubricants are used for urethral catheterisation chlorhexidine gel is widely used for this process however, there are no studies of the effects of lubricants the recurrence of urethral stricture. Future studies should look at the impact of different lubricants on the urethral stricture after EIU. The lubricants of the present study include a short following period and the absence of postoperative histological examination of the experimental and controls group. We did not exclusion the benign prostatic hyperplasia patients. The heterogeneity of the urethral stricture sites may be a limitation of the current study. And the absence of a power calculation can be a Institution. However, the present study is significant because the first prospective and randomized controlled study of the ability of HA to reduce the recurrence of urethral structure after EIU treatment. The long-term effects of HA installation during EIU should be confirmed by follow-up, and the histological effects should be by animal experimentation.

\section{CONCLUSIONS}

Visual Internal Urethrotomy is a simple and popular treatment for male stricture; however, the long term stricture-free rate is modest even after only a single procedure. The instillation of HA into the urethra during VIU decreased the incidence of urethral re-stricture after surgery without significant side effect.

\section{REFERENCES}

[1] Anger JT, Buckley JC, Santucci RA, et al. Trends in stricture management among male medicare beneficiaries: underuse of urethroplasty? Urology 2011;77(2):481-5.

[2] Bullock TL, Brandes SB. Adult anterior urethral strictures: a national practice patterns survey of board certified urologists in the United States. Journal of Urology 2007;177(2):685-90.

[3] Veeratterapillay R, Pickard RS. Long-term effect of urethral dilatation and internal urethrotomy for urethral strictures. Current Opinion in Urology 2012;22(6):467-73.

[4] Van Leeuwen MA, Brandenburg JJ, Kok ET, et al. Management of adult anterior urethral stricture disease: nationwide survey among urologists in the Netherlands. European Urology 2011;60(1):159-66.

[5] Granieri MA, Peterson AC. The management of bulbar urethral stricture disease before referral for definitive repair: have practice patterns changed? Urology 2014;84(4):946-9.

[6] Palminteri E, Maruccia S, Berdondini E, et al. Male urethral strictures: a national survey among urologists in Italy. Urology 2014;83(2):477-84.

[7] Ferguson GG, Bullock TL, Anderson RE, et al. Minimally invasive methods for bulbar urethral strictures: a survey of members of the American Urological Association. Urology 2011;78(3):701-6. 
[8] Dogra PN, Aron M, Rajeev TP. Core through urethrotomy with the neodymium: YAG laser for posttraumatic obliterative stricture of the bulbo membranous urethra. J Urol 1999;161(1):81-4.

[9] Ramanathan R. Intermittent self-catheterization versus regular outpatient dilatation in urethral stricture: a comparison: comment. Aust N Z J Surg 1999;69(12):884.

[10] Shirazi M, Khezri A, Samani SM, et al. Effect of intraurethral captopril gel on the recurrence of urethral stricture after direct vision internal urethrotomy: Phase II clinical trial. Int J Urpl 2007;14(3):203-8.

[11] Tabassi TK, Yarmohamadi A, Mohammadi S. Triamcinolone injection following internal urethrotomy for treatment of urethral stricture. Urol J 2011;8(2):132-6.

[12] Gall Y. [Hyaluronic acid: structure, metabolism and implication in cicatrisation]. Annales de Dermatologle et de Venerelogie 2010;(137 Suppl 1):S30-S9.

[13] Chung JH, Kang DH, Choi HY, et al. The effects of hyaluronic acid and carboxymethylcellulose in preventing recurrence of urethral stricture after endoscopic internal urethrotomy: a multicenter, randomized controlled, single-blinded study. Journal of Endourology 2013;27(6):756-62.

[14] Wolfram D, Tzankov A, Pülzl P, et al. Hypertrophic scars and keloids-a review of their pathophysiology, risk factors and therapeutic management. Dermatologic Surgery 2009;35(2):171-81.
[15] Jin T, Li H, Jiang LH, et al. Safety and efficacy of laser and cold knife urethrotomy for urethral stricture. Chin Med J (Engl) 2010;123(12):1589-95.

[16] Mundy AR. Management of urethral stricture. Postgrad Med J 2006;82(970):489-93.

[17] Holm-Nielsen A, Schultz A, Moller-Pecersen V. Direct vision internal urethrotomy. A critical review of 265 operation. Br J Urol 1984;56(3):308-12.

[18] Vicente J, Salvador J, Calfaratti J. Endoscopic urethrotomy versus urethrotomy plus Nd-YAG laser in the treatment of urethral stricture. Eur Urol 1990;18(3):166-8.

[19] Koc E, Arca E, Surucu B, et al. An open, randomized controlled, comparative study of the combined effect of intralesional traimcinclone acetonide and onion extract gel and intralesional triamcinolone acetonide alone in the treatment of hypertrophic scars and keloids. Dermatol Surg 2008;34(11):1507-14.

[20] Da-Silva EA, Sampaio FJ, Dornas MC, et al. Extracellular matrix changes in urethral stricture diseases. J Urol 2002;168(2):805-7.

[21] Hong JH, Choe JW, Kwon GY, et al. The effects of barrier materials on reduction of pericardial adhesion formation in rabbits: a comparative study of a hyaluronan-based solution and a temperature sensitive poloxamer solution/gel material. J Surg Res 2011;166(2):206-13. 